\title{
Multiple Variations of Branches of Abdominal Aorta
}

\author{
Salve V M , Ratanprabha C
}

Dept.Of Anatomy

Dr Pinnamaneni Siddhartha Institute Of Medical Sciences \& Reasearch Foundation,

Chinnaoutpalli, Gannavaram Mandal,

Krishna District, A.P. India

\section{CORRESPONDING}

Dr Vishal M. Salve

Associate Professor

Dept.Of Anatomy

Dr Pinnamaneni Siddhartha Institute Of Medical Sciences \& Reasearch Foundation,

Chinnaoutpalli, Gannavaram Mandal,

Krishna District, A.P, India

E-mail: salvevishal73@gmail.com

Citation

Salve V M, Ratanprabha C. Multiple Variation of Branches of Abdominal Aorta. Kathmandu Univ Med J 2011;33(1)72-6.

\begin{abstract}
:
The Abdominal aorta and its major branches supply oxygenated blood to nearly all the organs in the abdominal cavity. During routine dissection (January 2009) of a middle aged male cadaver at Dr. PSIMS, Gannavaram, Krishna Dist. (INDIA), the following variations of branches of abdominal aorta were found. The coeliac trunk gave off three branches. The first branch was left inferior phrenic artery which arose directly from coeliac trunk. The second branch bifurcates into left gastric artery and accessory hepatic artery for left lobe of liver. The second branch gave off splenic artery and common hepatic artery. The right testicular artery took its origin from right aberrant renal artery. This variation was associated with the presence of bilateral aberrant renal arteries for lower poles of both kidneys arising from abdominal aorta and aberrant renal arteries bilateral for upper poles originating from the renal arteries. Anatomical variation of testicular arteries is reported to be $4.7 \%$. Apart from creating hazards during abdominal surgery, vascular variation can also become a technical problem for infusion therapy and chemoembolisation of neoplasm in the liver.
\end{abstract}

\section{KEY WORDS}

Abdominal aorta, celiac trunk, aberrant renal arteries, accessory hepatic artery, inferior phrenic artery, splenic artery.

\section{INTRODUCTION:}

The Abdominal aorta and its major branches supply oxygenated blood to nearly all the organs in the abdominal cavity. ${ }^{1}$ The coeliac trunk supplies the abdominal oesophagus, stomach, duodenum up to major duodenal papilla, liver, pancreas, gall bladder and spleen. The common hepatic, splenic and left gastric arteries are considered as the "main classic branches" of the coeliac trunk.

Variations in the number of the renal arteries and their position with respect to the renal veins are common. ${ }^{2}$ Accessory renal arteries are commonly derived from the ipsilateral renal arteries, abdominal aorta, common iliac and superior mesenteric arteries. Rarely they originate from the external iliac, lumbar, ovarian, inferior mesenteric, superior suprarenal, inferior phrenic, right colic, subcostal, contralateral renal and splenic arteries or from the thoracic aorta. ${ }^{3}$

Usually inferior phrenic arteries arise from the abdominal aorta one on either side, just above the level of the coeliac trunk. They might originate by a common trunk from the aorta, the coeliac trunk or independently from the same sources. The inferior phrenic artery may arise from the renal, left gastric, superior mesenteric, suprarenal, or rarely from the common hepatic artery ${ }^{1}$.

The testicular artery usually arises from the anterolateral aspect of the abdominal aorta at the level of the second lumbar vertebra, 2.5 to $5 \mathrm{~cm}$ caudal to the renal artery. It may originate from the renal artery or as a branch from a suprarenal or lumbar artery .The testicular artery courses obliquely downwards and laterally behind the 
peritoneum into the pelvic cavity. ${ }^{3}$

Anatomical variations of testicular arteries with regard to their origin have been reported in $4.7 \%$ of cases. They were found to take origin either from unusually high level of aorta or from the renal artery. ${ }^{4}$ Bilateral aberrant renal arteries are found in 13- $16 \%$ of cases. ${ }^{5}$ Origin of right testicular artery from right renal artery is very rare. Unusual origin or course of the testicular artery may be of surgical importance in carrying out specific surgical procedures involving it or in diagnostic pathology related to testicular anatomy.

According to Williams et al the lateral splanchnic arteries, which are branches of the dorsal aorta at embryonic stage, persists bilaterally as testicular and three suprarenal arteries. ${ }^{6}$ Certain vascular and developmental anomalies of kidneys can be associated with variations in the course of the gonadal arteries. These anomalies are explained by the embryological development of both of these organs from the intermediate mesoderm of the mesonephric ridge or crest. Further the vasculature of kidneys and gonads are derived from the lateral splachnic branches of dorsal aorta.

While vascular anomalies are usually asymptomatic, they may become important in patients undergoing diagnostic angiography for gastrointestinal bleeding, coeliac axis compression syndrome, or prior to an operative procedure or transcatheter therapy.

Here we describe an unreported case of multiple variations of branches of abdominal aorta which includes variations of branches of coeliac trunk, bilateral aberrant renal arteries and right testicular artery branching from right aberrant renal artery for lower pole of right kidney in a middle aged cadaver.

\section{CASE REPORT:}

During routine dissection (January 2009) of a middle aged male cadaver at the Dr. PSIMS, Gannavaram, Krishna Dist. AP (INDIA), the following variations of branches of abdominal aorta were found. The coeliac trunk gave off three branches (Figure 1). The first branch was left inferior phrenic artery which arose directly from coeliac trunk. The second branch trifurcated into two left gastric arteries and one accessory hepatic artery for left lobe of liver. The third branch gave off splenic artery and common hepatic artery. The common hepatic artery gave off hepatic artery which divided into two branches before entering right lobe of liver. The right testicular artery was found to arise from right aberrant renal artery for the lower pole of the right kidney (Figure 3).This variation was associated with the presence of bilateral aberrant renal arteries for lower poles of both the kidneys arising from abdominal aorta and bilateral aberrant renal arteries for upper poles originating from the renal arteries. Right aberrant renal artery for lower pole originated from front of the abdominal aorta at the level of second lumbar vertebra. Right testicular artery was noted to arise from right aberrant renal artery for lower pole about $0.5 \mathrm{~cm}$ from its origin. Right aberrant renal artery for upper pole originated from right renal artery about $1 \mathrm{~cm}$ away from medial border of the right kidney. Right renal artery took its origin from abdominal aorta just below the origin of coeliac trunk at the level of upper border of first lumbar vertebra. Left aberrant renal artery for lower pole originated from front of the abdominal aorta at the level of first lumbar vertebra just below the origin of the left renal artery. Left testicular artery was found to arise from abdominal aorta. Left aberrant renal artery for upper pole arose from left renal artery about $1 \mathrm{~cm}$ away from medial border of the left kidney. Left renal artery took origin from abdominal aorta at the level of L1 vertebra. In the same case, iliolumbar artery was noted to be a direct branch from common iliac artery. However, the abdominal organs revealed no anomalies.

\section{DISCUSSION:}

The abdominal aorta is the major artery of abdomen which bifurcates into right and left common iliac arteries. ${ }^{7}$ The coeliac trunk is the chief artery of the foregut. It supplies all derivatives of the foregut that lie in the abdominal cavity. It arises from the ventral portion of the abdominal aorta at the level of T12/L1 as a single trunk being $1.5-2 \mathrm{~cm}$ in length. It gives three main branches as left gastric, common hepatic and splenic arteries.

Cavdar et al reported a case, in which the left inferior phrenic artery and the left gastric artery arose from the long coeliac trunk $(4.3 \mathrm{~cm})$ via a common trunk. ${ }^{8}$

Kuo-Hsein Chiang et al studied 405 patients angiographically for evaluation of hepatic artery variations. Single accessory

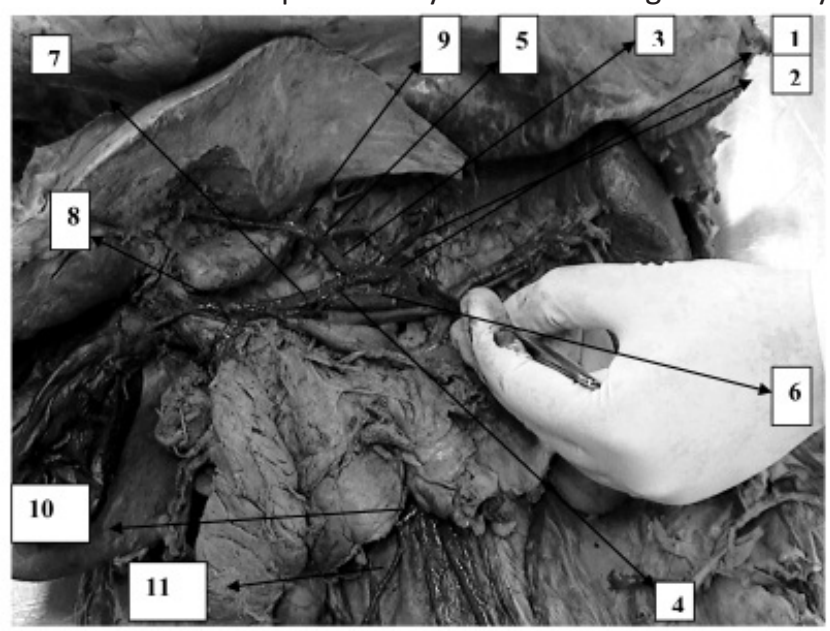

Figure 1. Dissection of abdomen showing the anomalous branching pattern of abdominal aorta. (1. Coeliac trunk, 2. Left inferior phrenic artery, 3. Branch-2 from celiac trunk which gives of two left gastric and accessory hepatic arteries, 4. Branch-3 from celiac trunk which gives of two splenic and common hepatic arteries, 5. Left gastric arteries, 6. Splenic artery, 7. Common hepatic artery, 8. Hepatic artery, 9. Accessory hepatic artery, 10. Right aberrant renal artery for lower pole \& 11 . Right testicular artery) 


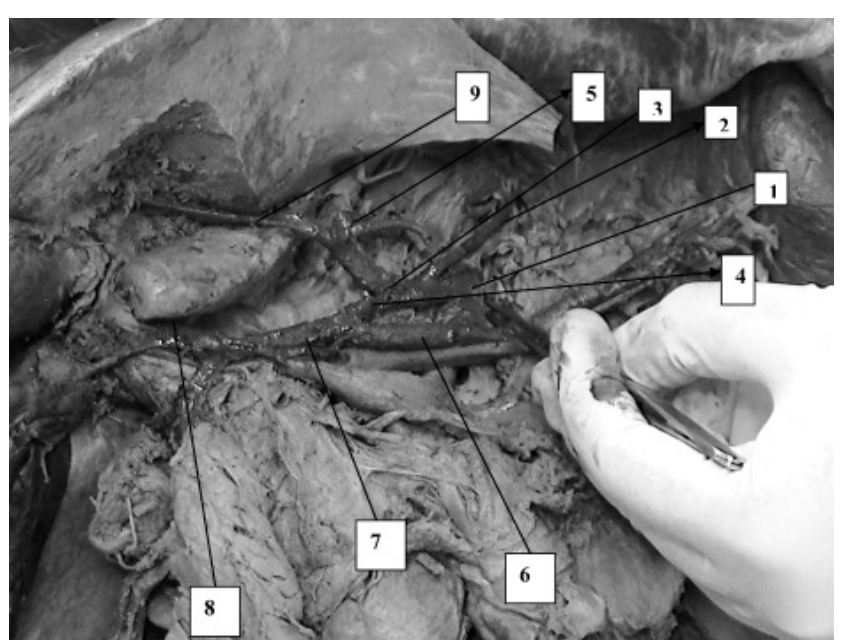

Figure 2. Dissection of abdomen showing the anomalous branching pattern of coeliac trunk. (1. Coeliac trunk, 2. Left inferior phrenic artery, 3. Branch-2 from celiac trunk which gives of two left gastric and accessory hepatic arteries, 4. Branch-3 from celiac trunk which gives of two splenic and common hepatic arteries, 5. Left gastric arteries, 6. Splenic artery, 7. Common hepatic artery, 8. Hepatic artery \& 9. Accessory hepatic artery)

hepatic artery was found in $28.1 \%$ (114) of cases. ${ }^{9}$ More than two hepatic arteries were found in $2.0 \%$ cases. Seventeen patterns origin of accessory hepatic artery were identified in this study. Some of the important patterns were accessory hepatic artery as direct branch from coeliac

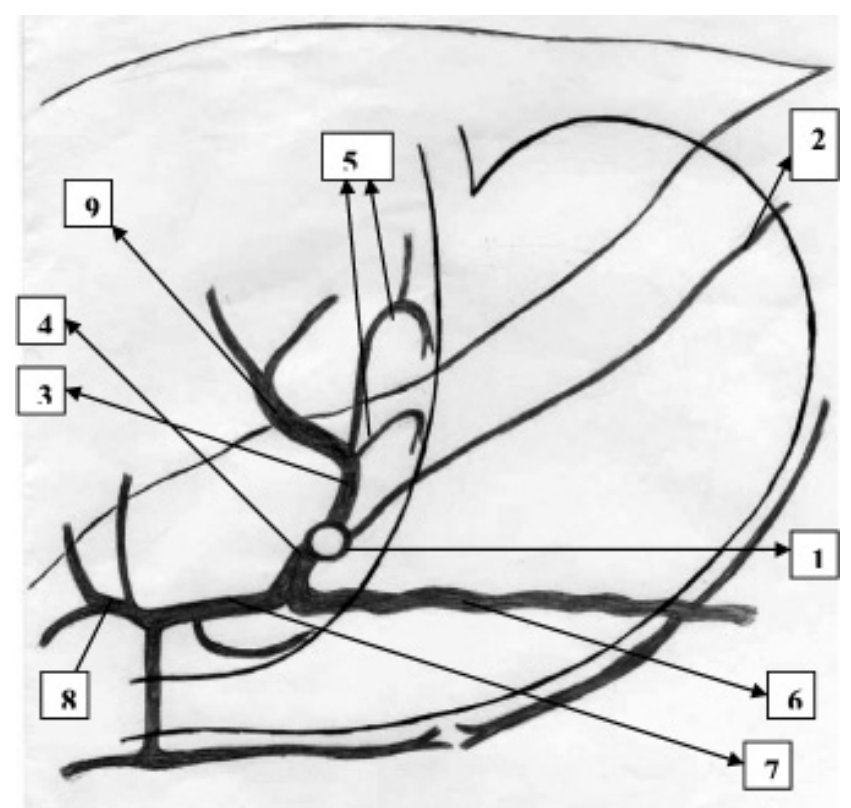

Figure 2a. Schematic diagram showing variations branches of celiac trunk. (1. Coeliac trunk, 2. Left inferior phrenic artery, 3. Branch-2 from celiac trunk which gives of two left gastric and accessory hepatic arteries, 4. Branch-3 from celiac trunk which gives of two splenic and common hepatic arteries, 6. Splenic artery, 7. Common hepatic artery, 8. Hepatic artery \& 9. Accessory hepatic artery) trunk, branch from common hepatic artery, left gastric artery etc.

Piano et al stated that the right and left inferior phrenic arteries occasionally originated as a common trunk from the aorta, coeliaco-mesenteric system or adreno-renal system. ${ }^{10}$ He observed that inferior phrenic arteries were usually paired ( left and right) and their origin were summarized as follows: a) the abdominal aorta itself ( $61.6 \%$ ), b) ventro-visceral arteries ( coeliaco-mesenteric system of aorta) including the coeliac trunk (28.2\%), and left gastric artery $(2.9 \%)$, c) the latero-visceral arteries ( adreno-renal system of the aorta) including the middle adrenal artery (2.9\%), and renal artery (4.3\%).

Ugur Ozkan et al in his angiographic evaluation of origin and variation of renal arteries (163 females \& 692 males), found renal arteries originated between the first and second lumbar vertebral level in most patients. ${ }^{5}$ Variations of renal arteries included multiple arteries in $24 \%$, bilateral multiple arteries in 5\%, and early division in $8 \%$ of cases. Additional renal arteries on the right side were found in $16 \%$ and on the left side in $13 \%$ of cases. This result should

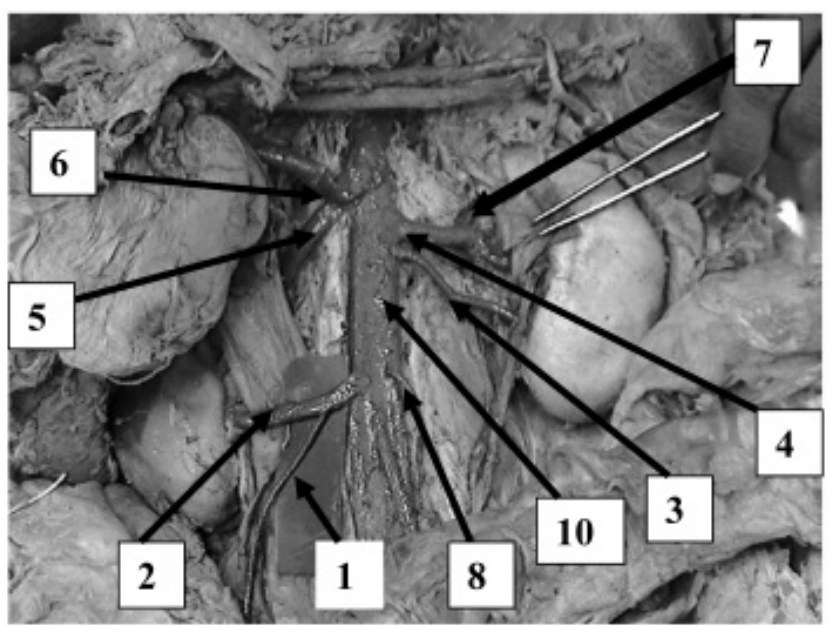

Figure 3. Dissection showing right and left renal, testicular and aberrant renal arteries. (1. Right testicular artery, 2. Right aberrant renal artery for lower pole, 3 . Left aberrant renal artery for lower pole, 4. Left renal artery, 5. Right renal artery, 6. Coeliac trunk, 7. Left aberrant renal artery for upper pole, 8. Left testicular artery, 9. Right aberrant renal artery for upper pole \& 10. Abdominal aorta)

be kept in mind when a non-invasive diagnostic search is performed for renal artery stenosis or when renal surgery related to renal arteries is performed.

According to Notkovich, the gonadal arteries have been classified into three types based on their anatomical relationship to the renal vein: Type I-gonadal arteries arising from the renal arteries behind or below the renal veins and passing downwards and laterally into inguinal canals, Type II - gonadal arteries arising from the aorta at the level of the renal veins and crossing in front of them, Type III - gonadal arteries arising from the aorta behind or below the renal 


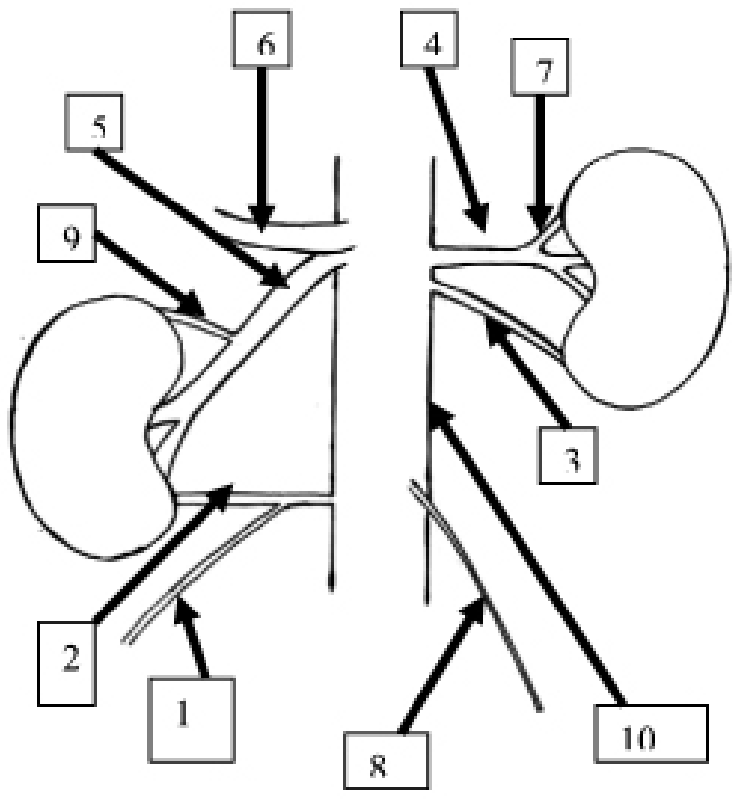

Figure 3a. Schematic diagram showing right and left renal, testicular and aberrant renal arteries. (1. Right testicular artery, 2. Right aberrant renal artery for lower pole, 3 . Left aberrant renal artery for lower pole, 4. Left renal artery, 5. Right renal artery, 6. Coeliac trunk, 7. Left aberrant renal artery for upper pole, 8. Left testicular artery, 9. Right aberrant renal artery for upper pole \& 10. Abdominal aorta)

veins and course upwards to arch over the renal veins. ${ }^{11}$

Pai and Vadgaokar in their study found variations of testicular arteries in $14.7 \%$ (34 adult male cadavers aged 40-60 years) of cases. ${ }^{12}$ The following variations were seen in their study:

Variation 1- The testicular artery was a branch of aberrant renal artery for lower pole of kidney. This anomalous pattern was seen in five cadavers $(7.4 \%)$, out of which three were on the right side and two on the left.

Variation 2- The testicular artery was a branch of the renal artery. This anomalous pattern was seen on the right side of only one cadaver (1.4\%).

Variation 3- High origin of testicular artery. This anomalous pattern was seen on the left sides of three cadavers (4.4\%).

Variation 4- Double testicular artery. This anomalous pattern was seen on the right side of only one cadaver (1.4\%).

Ishiqami $\mathrm{K}$ et al studied 118 patients with conventional angiography for detection of an accessory left gastric artery. ${ }^{13}$ Result of this study revealed an accessory left gastric artery in 25 (21.2 \%) of 118 cases. According to Hollinshead W.H. ${ }^{7}$ an accessory left gastric artery is present in about $11.5 \%$ of population. It arises from aorta independently in about $4.5 \%$ of population. An accessory right gastric artery is present in about $1.45 \%$ of population.

The anatomical variations of the coeliac trunk are due to unusual embryological development of the ventral splanchnic branches of the aorta. ${ }^{8}$ In our cases, the variations of the coeliac trunk may be due to same embryological cause. The formation of the aorta begins during the third week of embryological development. Many segmental arteries arise from the primitive dorsal aorta. As the embryo continues to develop, most of the segmental arteries regress, except for the precursor of the segmental arteries to the three major mesenteric vessels. The $10^{\text {th }}$ segmental artery gives rise to coeliac trunk. ${ }^{1}$ The variations in these arteries arise from differences in the pattern of the partial disappearance or the survival of the ventral splanchnic arteries and ventral longitudinal channel. ${ }^{14}$

The developmental origins of testicular blood vessels are very complex. Nine lateral mesonephric arteries are divided into the cranial, middle and caudal group. One of the caudal arteries usually persists and differentiates into the definitive gonadal artery. The persistence of cranial lateral mesonephric artery results in a high origin of the gonadal artery, probably from suprarenal artery or from a more superior aortic level. Persistence of more than one lateral mesonephric arteries result in double, triple or quadruple gonadal arteries. If the kidney ascends much higher carrying its renal vein to a higher level than the origin of gonadal artery, the latter will be forced to follow an arched course around the renal vein. ${ }^{12,15}$

In our opinion, such arterial variations as noted in the present study should not be ignored during abdominal operative procedures. Many complications could be avoided with the accurate knowledge of such arterial variations of branches of abdominal aorta. Knowledge of such variations will play a significant role in carrying out surgical intervention safely in the abdomen and also in the interpretation of angiographic reports. Vascular variations can also become a technical problem for infusion therapy and chemoembolisation of neoplasm in the liver.

\section{REFERENCES}

1. Lin P.H., Chaikof E.L. Embryology, anatomy and surgical exposure of the great abdominal vessels. Surg. Chin. North Am. 2000 Feb; 80(1): 417-33.

2. Jeffery R. Unusual origin of renal arteries. Radiol. 1972; 102:302.

3. Skeys D. The arterial supply of the human kidney with special references to accessory renal arteries. Brit. J. Surg.1963; 50:668.

4. Samuel Asala, Shyama C. Chaudhary M. Bidmos. Anatomical variations in the human testicular blood vessels. Annals of anatomy Anatomischer Anzeiga. 2001 Nov; 183(6): 545-549.

5. Ugur Ozkan, Levent Oguzkurt. Renal artery origin and variations: angiographic evaluation of 855 consecutive patients; Diagnostic Interventional Radiology 2006; 12:183 -86.

6. Williams P L, Warwick R, Dyson M, Bannister LH. Gray's Anatomy 37th edition (Angiology). Churchill Livingstone. 1989; 764, 773 - 76 
7. Hollinshead W.H. Anatomy for surgeons. Vol: 2 (The Thorax, Abdomen and Pelvis, The stomach, duodenum, pancreas and spleen), 2nd Ed. New York, Harper \& Row Publisher, 1961:590.

8. Chiang K, Chang P, Lee P, Ling C, Lee W, Lee C, Chou S. Angiographic evaluation of hepatic artery variations in 405 cases. Chin J Radiol 2005; 30:75-81.

9. Cavder S, Sehirii U, Pekin B. Celiacomesenteric trunk. Clin. Anat. 1997; 10: 231-234.

10. Piano DX, Ohtsuka A, Murakami T. Typology of abdominal arteries, with special references to inferior phrenic arteries and their oesophagial branches. Acta Med Okayama 1988; 52: 189-96.

11. Notkovich $\mathrm{H}$. Variation of testicular and ovarian arteries in relation to the renal pedicle. Surg Gynecol Obstet 1956; 103:487-95.
12. Pai MM, Vadagaonkar R, Rai R, Nayak SR, Jiji RJ, Ranade A, Prabhu LV, Madhyarstha $S$. A cadaveric study of the testicular artery in the south Indian population. Singapore Med J 2008; 49(7): 551.

13. Ishiqami K, Yoshimitsu K, Irie H, Tajima T, Asayama $Y$, Hirakawa M, Honda $\mathrm{H}$. Accessory left gastric artery from left hepatic artery shown on MDCT and conventional angiography. AJR Am J. Roentgenol 2006; 187(4):1002-1009.

14. Oswa $T$, Feng $X$, Sasaki N, Nagato $S$, Matsumoto $Y$, Onodera $M$, Nara E, Fujimura A, Nozaka Y. Rare case of the inferior mesenteric artery and the common hepatic artery. Clinical Anatomy 2004; 17:518-521.

15. Moore KL \& Persaud TVN. The Developing Human (Clinically Oriented Embryology) 7th edition. (The cardiovascular system) Saunders. 2003; 335. 